\title{
COMPARISON OF FILTERING AND CLUSTERING TECHNIQUES IN DIAGNOSIS OF INFANTS RETINOPATHY RISK
}

\author{
Niousha Hormozi ${ }^{1}$, Seyed Amirhassan Monadjemi ${ }^{2}$ and Gholamali \\ Naderian $^{3}$ \\ ${ }^{1}$ Faculty of Computer Engineering, University of Isfahan, Isfahan, Iran \\ ${ }^{2}$ Faculty of Computer Engineering, University of Isfahan, Isfahan, Iran \\ ${ }^{3}$ Isfahan University of Medical Sciences, Isfahan, Iran \\ ${ }^{1}$ Niousha.hormozi@yahoo.com, ${ }^{2}$ amonadjemi@yahoo.co.uk, \\ 3 naderianuseyahoo.com
}

\begin{abstract}
ROP is an eye disease in premature infants. In infants who are born earlier than normal, retinal vessel growth stops. Early treatment is very crucial in this case as it can end up to blindness if the diagnosis has not done in a short time. The purpose of this research is to design an intelligent automated system for the early detection of disease at an early stage to prevent these babies from dangerous consequences. In this study, we analyzed the images in the Lab color space, and evaluated the efficiency of applying filters named, Canny Laplacian and Sobel. The results indicate relatively higher efficiency and quality of the Laplacian filter in ROP diagnosis.
\end{abstract}

\section{KEYWORDS}

Retinopathy of prematurity, Canny filters, Laplacian filters, Sobel operator, Lab color space, ROP

\section{INTRODUCTION}

ROP is an eye disease in premature infants which is due to the abnormal growth of retinal blood vessels that leads to scarring in the retina eventually. The main cause of visual impairment and blindness in ROP is retinal detachment which is the subsequence of the scars in turn. Swollen and twisted veins contribute to Plus-disease (PD) that is considered as the most severe type of ROP[4]. In Figure 1, retinal detachment of scleral membrane due to the stretching of the wound contraction caused by abnormal blood vessel growth is shown. 


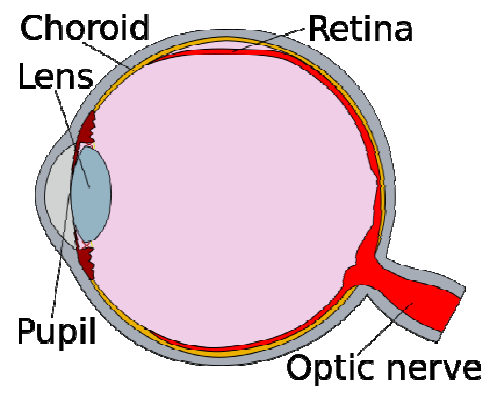

Figure 1. Retinal detachment caused by abnormal vessel growth [1].

In infants born earlier than normal (premature infants) the growth of retinal vessel stops before the retinal surface can completely be covered by retinal vessels. So the uncompleted sections would be unable to receive enough oxygen and food by blood circulation that consequently causes the disease. It is more common in infants weighing less than $1500 \mathrm{gr}$ and gestational age less than 31 weeks[5]. Thus, the ROP normal growth of blood vessels stops and abnormal blood vessels grow, if left untreated it can lead to vision loss or blindness. Severity of ROP is determined by following factors:

1- Which area the new vessels have been located. Figure 2 illustrates the defined areas.

2- How much retinal vessel network is grown?

3- How much swollen the veins are?

4- The presence or absence of plus-disease.

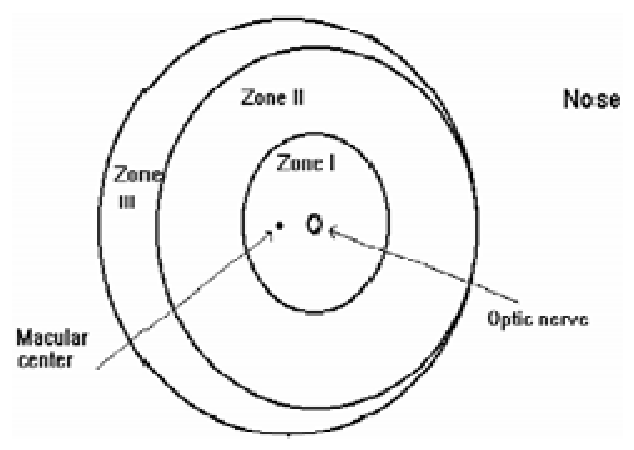

Figure 2. Defined areas of the eye [1]

Structural changes in the vessels can be studied in several ways:

- Examining by ophthalmoscope directly

- By examining images of the retina

Ophthalmoscope is an instrument that helps the physician so that they can see a perspective of the retina, it is challenging to use though, due to the need for expertise and experience. Figure 3 displays a typical ophthalmoscope. 


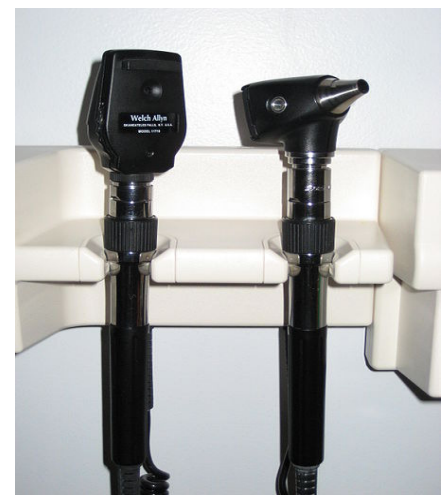

Figure 3. Ophthalmoscope[2].

So it is important to have a system that can be used to increase the accuracy of the examination done by physicians or practitioners (e.g. by replacing trained nurses). Figures 4, 5 and 6 show abnormal growth, tortuosity of vessels and scarring of the retina. Given these figures, it seems it is possible to extract vessels automatically and use it to diagnose ROP.

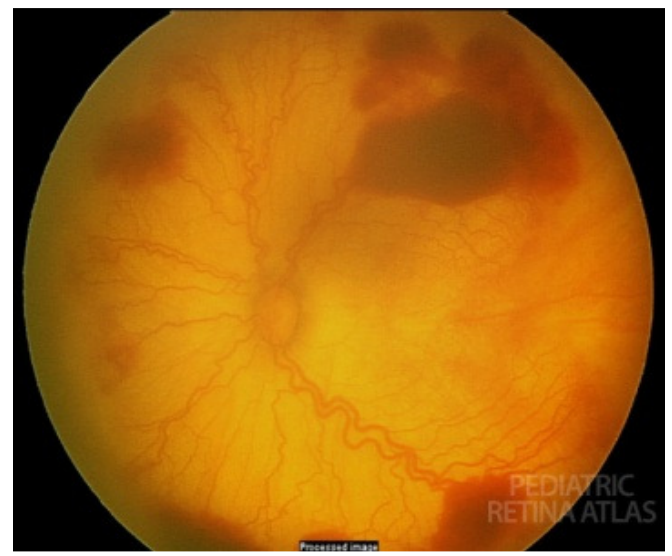

Figure 4. Retinal vessel tortuosity and scars

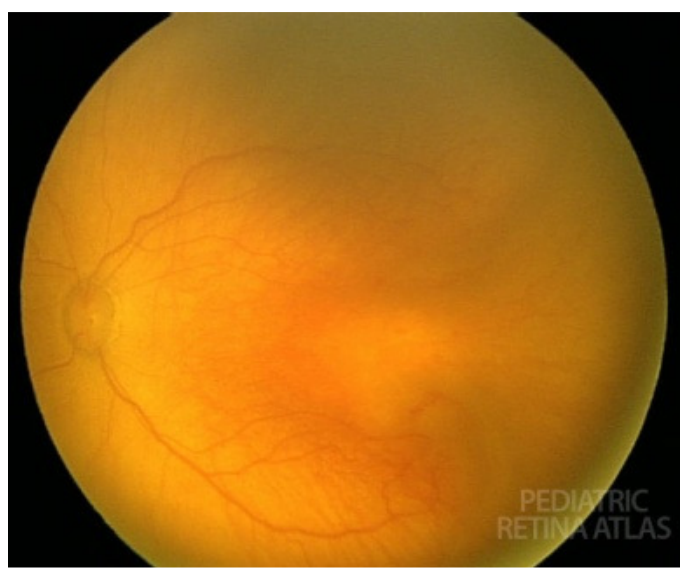

Figure 5 Incomplete vascular growth 


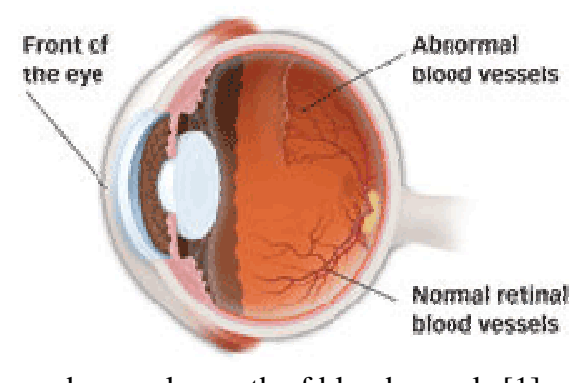

abnormal growth of blood vessels [1]

Factors that affect vessel segmentation are as follows:

- Vessel's width, shape and colour are not the same. Vessel's widths differ from one pixel to 12 pixels.

- There are other structures in images similar to vessel structures, such as retinal disc boundary, or nerves.

- Crossing points and bifurcations could confuse the techniques.

- Edge of the disk may be classified as vessel incorrectly.

- Sometimes the local contrast between the vessels and the background is very low. Particularly, thin veins have less contrast with their background $[4,6]$.

Despite many successful works in the field of image processing techniques performed on adult retinal images, most of these techniques fail when it comes to infants images. That's because several parameters are different in adults and infants images, such as resolution of images, blood vessels thickness and noise ratio. Vessel detection in images is more difficult in infants than in adults; however, both are equally important issues[7]. Figure 7 and 8 show structural differences between the infants and adult images.

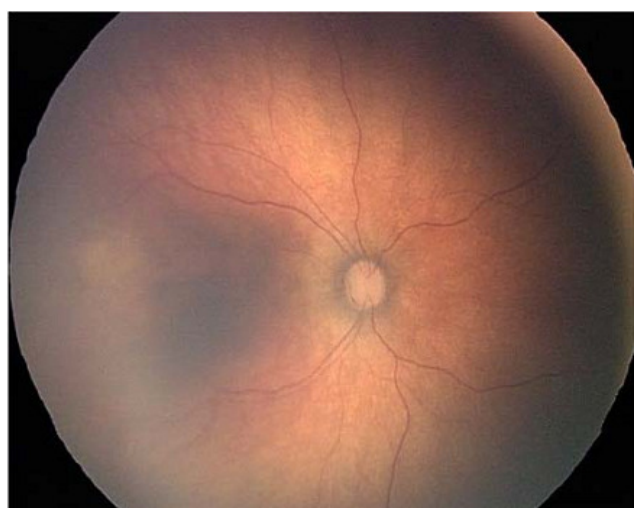

Figure7. An examples of infant retinal image 


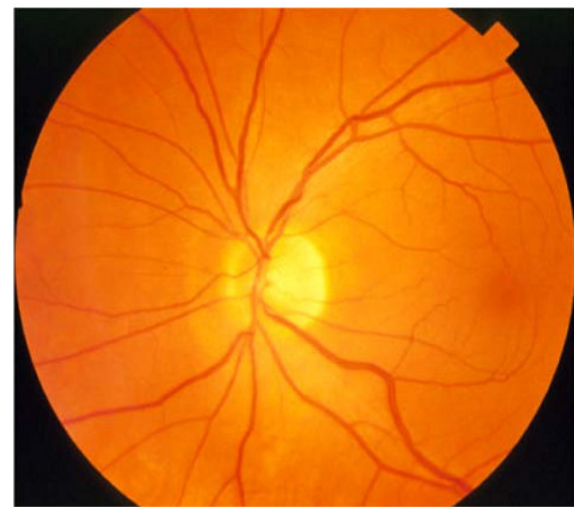

FIGURE 8. AN EXAMPLE OF ADULT RETINAL IMAGE [3].

\section{MATERIALS AND METHODS}

In this paper, the image is segmented in Lab color space. For this, clustering is applied on channels $\mathrm{a}$ and $\mathrm{b}$ in the Lab color space. The number of clusters are considered two, constituting of veins and background. After separating the gray segment, the ratio of the number of pixels in gray segment is evaluated to the total pixels in the image. This represents the area in which vessels are not fully grown and need regular checkups to ensure that it is fully developed in time and if not, the urgent treatments are needed to help the vascular structure grow normally. Figures 9, 10 and 11 show the clustering. The original image can be seen in Figure 9. Figure 10 and 11 represent the clusters including the background and the vessels respectively. The second cluster of pixels ratio to the total number of image pixels demonstrates the performance of this function in isolating the area of the vessels. In figure 11 this ratio was 2.17.

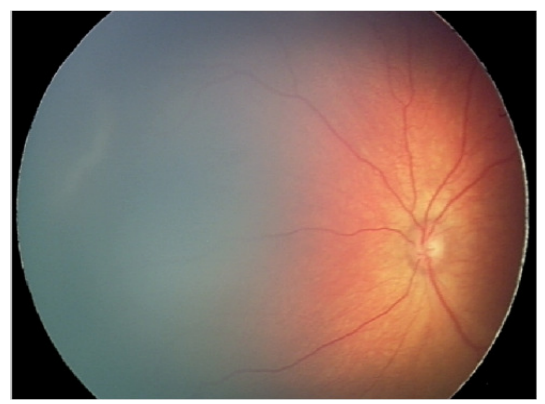

Figure 9. The original image

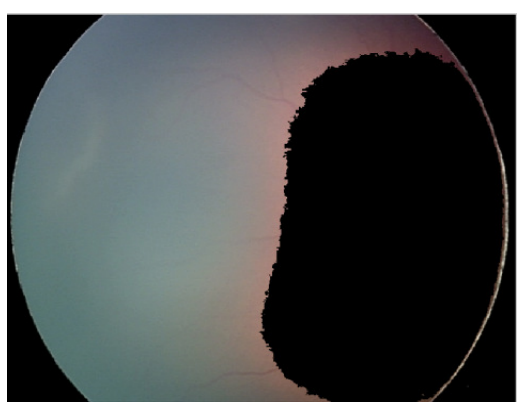

Figure 10. The first cluster, consisting undeveloped vessels 


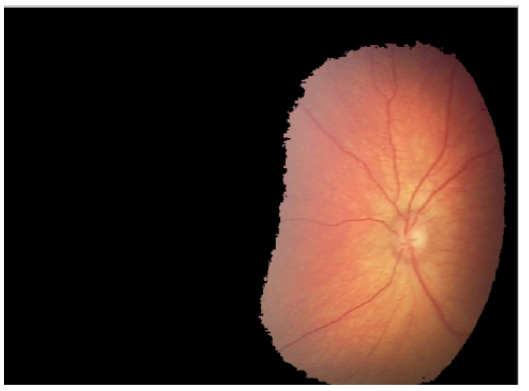

Figure 11. the second cluster with the ratio of 2.17

Table 1 illustrates the results of applying a threshold 0.5 to the ratios in terms of accuracy, specificity and sensitivity.

Table1. The result of thresholding the ratios with threshold 0.5

\begin{tabular}{|c|c|c|}
\hline Sensitivity & Specificity & Accuracy \\
\hline$\% 74$ & $\% 50$ & $\% 69$ \\
\hline
\end{tabular}

In this paper, we also used the three operators named; Canny, Laplacian and Sobel operator to compare the results of edge detection in finding vessels the performance of these three are shown in Figures 12 to 18. After applying Laplacian operator, two different morphological operators used for the sake of noise removal; morphological opening with $3 \times 3$ squares and lines with a difference of 15 degrees $(0,15,30,60,75,90,105,120,135,150,165)$ and 17 pixels length.

Laplacian filter can be seen in Figure 12, it has been accurately able to isolate the vessel. The morphological operators are used to remove noise from the image.

Figure 13 shows the result of opening morphological operator on Figure 12.

Figure 14 shows the result of applying lines 15 degree difference lines of length 12 to open Figure 12.

Figure 15, 16 are the result of applying Sobel and Canny operators respectively.

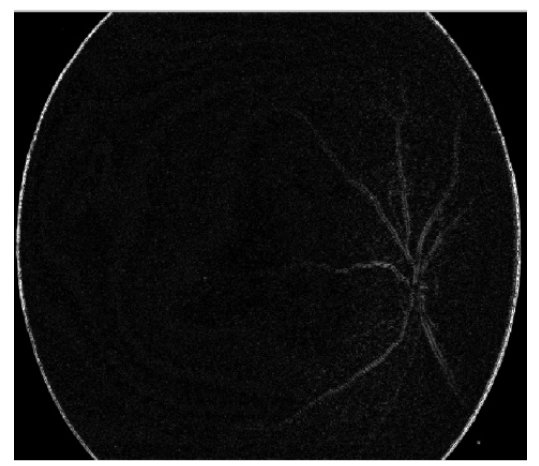

Figure 1. The result of applying Laplacian filter 


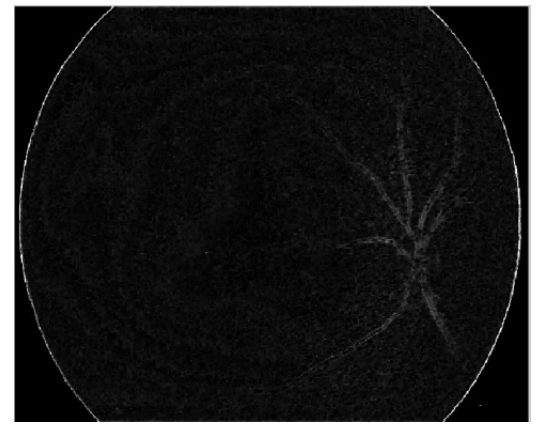

Figure 13. The result of opening by the 33 square

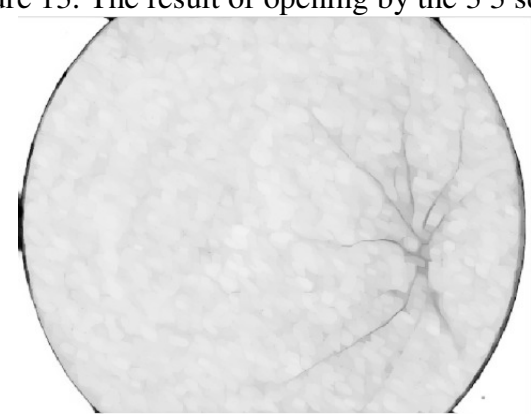

Figure 14. The result of opening by lines with a difference of 15 degrees

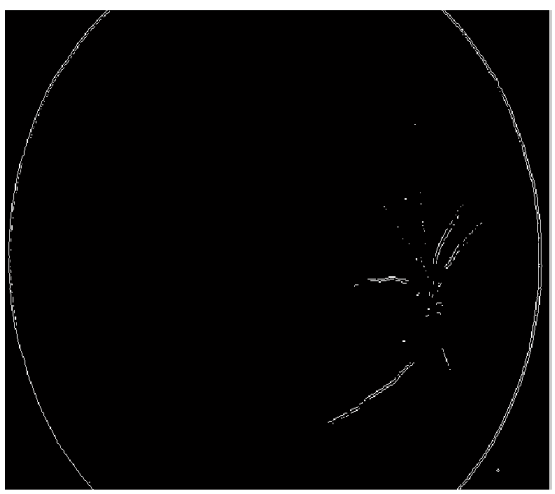

Figure 15. The result of applying Sobel operator

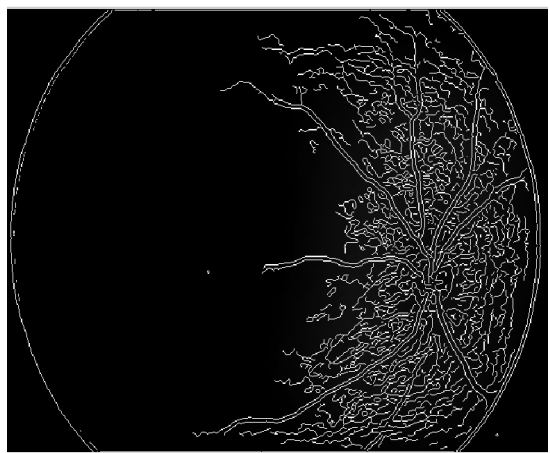

Figure16. The result of Canny operator 


\section{CONCLUSIONS}

In this paper we compared methods of edge detection for extracting vessels. We also segmented the retinal vessels in Lab color space images. It is conducted from the results that noise is removed followed by Laplacian filters as well as the opening operator on Laplacian filtered images of the retina. Thus, the detection of retinal vessel network provides determining the possibility of ROP risk. This plays an important role for newborn infants who born with undeveloped retinal vessels to be followed in a regular basis so that it would prevent them from blindness in future.

\section{REFERENCES}

[1] Available: http://en.wikipedia.org/w/index.php?title=Retinopathy_of_prematurity\&oldid=621118923

[2] facts about retinopathy of prematurity (ROP)," National Eye Instiute, october 2009. [Online]. Available: http://www.nei.nih.gov/health/rop/rop.asp. [Accessed 2012 may 14]. .

[3] K. A. Vermeer, F. M. Vos, H. Lemij, and A. M. Vossepoel, "A model based method for retinal blood vessel detection," Computers in Biology and Medicine, vol. 34, pp. 209-219, 2004.

[4] L. Gang, O. Chutatape, and S. M. Krishnan, "Detection and measurement of retinal vessels in fundus images using amplitude modified second-order Gaussian filter," Biomedical Engineering, IEEE Transactions on, vol. 49, pp. 168-172, 2002.

[5] D. K. Wallace, "Diagnostic and Treatment Advances in Retinopathy of Prematurity," 2007.

[6] M. Sofka and C. V. Stewart, "Retinal vessel centerline extraction using multiscale matched filters, confidence and edge measures," Medical Imaging, IEEE Transactions on, vol. 25, pp. 1531-1546, 2006.

[7] L. Sukkaew, B. Uyyanonvara, S. S. Makhanov, S. Barman, and P. Pangputhipong, "Automatic tortuosity-based retinopathy of prematurity screening system," IEICE transactions on information and systems, vol. 91, pp. 2868-2874, 2008. 